\title{
Alternative fuels in the well-to-wheel debate
}

Patrick R. Schmidt, Ludwig-Bölkow-Systemtechnik GmbH, Prof. Dr. Kurt Kirsten, Co-Author: Prof. Dr. Jens Hadler, both APL Automobil-Prüftechnik Landau $\mathrm{GmbH}$

Zum Zeitpunkt der Drucklegung lagen diese Unterlagen noch nicht vor. Wir bitten um Verständnis. 